\title{
ON THE CONSISTENCY OF THE \\ TWO-SAMPLE EMPTY CELL TEST
}

\author{
M. Csorgo ${ }^{*}$ and Irwin Guttman
}

(received August 15, 1962)

1. Introduction. This paper considers the consistency of the two-sample empty cell test suggested by S. S. Wilks [2]. A description of this test is as follows: Let a sample of $n_{1}$ independent observations be taken from a population whose cumulative distribution function $F_{1}(x)$ is continuous, but otherwise unknown. Let $\mathrm{x}_{(1)}<\mathrm{x}_{(2)}<\ldots<\mathrm{x}_{\left(\mathrm{n}_{1}\right)}$ be their order statistics. Let a second sample of $\mathrm{n}_{2}$ observations be taken from a population whose cumulative distribution function is $F_{2}(x)$, assumed continuous, but otherwise unknown.

$$
\text { Define cells } I_{1}, \ldots, I_{n_{1}+1} \text { by }
$$

$$
I_{i}=\left(X_{(i-1)}, X_{(i)}\right], \quad i=1, \ldots, n_{1}+1
$$

where $x_{(0)}=-\infty$ and $x_{\left(n_{1}+1\right)}=+\infty$.

Let $r_{1}, \ldots, r_{n_{1}+1}$ be the number of observations of the second sample that lie in $I_{1}, \ldots, I_{n_{1}+1}$ respectively. Let $S_{0}$ be the number of $I_{i}, i=1, \ldots, n_{1}+1$ which are

\footnotetext{
* Work supported by a National Research Council of Canada Studentship.
}

Canad. Math. Bull. vol. 7, no. 1, January 1964 
such that $r_{i}=0$, that is, the number of empty cells. Under the hypothesis that $F_{1}=F_{2}$, Wilks in [2] and [3] gives a somewhat complicated analytic derivation of the probability function of $S_{0}$ and obtains the result

$$
P\left(S_{0}=s_{0}\right)=\frac{\left(\begin{array}{c}
n_{1}+1 \\
s_{0}
\end{array}\right)\left(\begin{array}{c}
n_{2}-1 \\
n_{1}-s_{0}
\end{array}\right)}{\left(\begin{array}{c}
n_{1}+n_{2} \\
n_{1}
\end{array}\right)}=p\left(s_{0}\right)
$$

where the sample space of $S_{0}$ is given by

$$
f=\left[k, k+1, \ldots, n_{1}\right] \text { and } k=\max \left[0, n_{1}+1-n_{2}\right]
$$

A simplified proof of (1.2) may be found in [4].

Using (1.2), it can be easily shown that

$$
\begin{gathered}
E\left(S_{0}\right)=\frac{n_{1}\left(n_{1}+1\right)}{n_{1}+n_{2}} \\
\sigma^{2}\left(S_{0}\right)=\frac{n_{1}^{2}\left(n_{1}^{2}-1\right)}{\left(n_{1}+n_{2}\right)\left(n_{1}+n_{2}-1\right)}+\frac{n_{1}\left(n_{1}+1\right)}{n_{1}+n_{2}}-\frac{n_{1}^{2}\left(n_{1}+1\right)^{2}}{\left(n_{1}+n_{2}\right)^{2}}
\end{gathered}
$$

(For these results see Wilks [2] and [3] where the method of factorial moments is used to obtain them.)

$$
\begin{aligned}
& \text { If we let } n_{2}=\rho n_{1}+O(1), \rho>0, \text { the se reduce to } \\
& E\left(S_{0}\right)=n_{1}\left(\left(\frac{1}{1+\rho}\right)+O\left(\frac{1}{n_{1}}\right)\right)
\end{aligned}
$$

$$
\sigma^{2}\left(S_{0}\right)=n_{1}\left(\frac{p^{2}}{(1+\rho)^{3}}+O\left(\frac{1}{n_{1}}\right)\right)
$$


which in turn imply that

$$
E\left(\frac{s_{0}}{n_{1}+1}\right) \rightarrow \frac{1}{1+\rho}
$$

and

$$
\sigma^{2}\left(\frac{s_{0}}{n_{1}+1}\right) \rightarrow 0
$$

as $\mathrm{n}_{1}, \mathrm{n}_{2} \rightarrow \infty$, and by Tchebychev's inequality, the se results imply that $S_{0} / n_{1}+1$ converges in probability to $\frac{1}{1+\rho}$, if $F_{1}=F_{2}$

We can use these results to make a test of the hypothesis $F_{1}=F_{2}$ at the approximate $100 \alpha \%$ level. This is given by

$$
\left\{\begin{array}{l}
\text { Reject if } s_{0} \geq b \\
\text { Accept otherwise }
\end{array}\right.
$$

where $b$ is such that

$$
\mathrm{P}\left(\mathrm{S}_{\mathrm{o}} \geq \mathrm{b}\right)=\sum_{\mathrm{s}_{\mathrm{o}}=\mathrm{b}}^{\mathrm{n}_{1}} \mathrm{p}\left(\mathrm{s}_{\mathrm{o}}\right) \leq \alpha
$$

(1.6)

$$
P\left(S_{0} \geq b-1\right)=\sum_{s_{0}=b-1}^{n_{1}} p\left(s_{0}\right)>\alpha .
$$

Tables of (1.6) have been tabulated by the authors for $\alpha=.01$ and .05 and published in Technometrics [4].

2. Consistency. The form of the test (1.5) follows from the following considerations. Let $G_{0}$ be the class of pairs of 
continuous cumulative density functions $\left(F_{1}(x), F_{2}(x)\right)$ such that $F_{1}(x)=F_{2}(x)$. Let $F_{1}^{-1}(u)$ be the inverse of the c.d.f. $F_{1}(x)$ and let $G_{1}$ be the class of pairs of continuous c.d.f.'s $\left(F_{1}(x), F_{2}(x)\right)$ satisfying:

(i) $F_{2}\left(F_{1}^{-1}(u)\right)$ has a derivative, say $g(u)$, for all $u$ on $(0,1)$ except possibly for a set of probability measure zero.

(ii) The derivatives of $F_{2}\left(F_{1}^{-1}(u)\right)$ and $F_{1}\left(F_{1}^{-1}(u)\right)=u$ with respect to $u$ on $(0,1)$ differ over a set of positive probability.

In [3] Wilks states the following

THEOREM. The test defined by (1.5) and (1.6) is consistent for testing any $\left(F_{1}, F_{2}\right) \in G_{0}$ against any $\left(F_{1}, F_{2}\right) \in G_{1}$ as $\mathrm{n}_{1}, \mathrm{n}_{2} \rightarrow \infty$ so that $\mathrm{n}_{2}=\mathrm{n}_{1} \rho+O(1)$, where $\rho>0$.

To prove this theorem it is sufficient to show that if $\left(F_{1}, F_{2}\right) \in G_{1}, S_{0} /\left(n_{1}+1\right)$ converges in probability to a number greater than $1 /(1+\rho)$ as $n_{1}, n_{2} \rightarrow \infty$ with $\frac{n_{2}}{n_{1}} \rightarrow \rho>0$, for it will be recalled from $(1.4)$ that $1 /(1+\rho)$ is the quantity to which $S_{0} /\left(n_{1}+1\right)$ converges in probability if $\left(F_{1}, F_{2}\right) \in G_{0}$.

We recall that $r_{1}, \ldots, r_{n_{1}+1}$ denote the number of observations of the second sample that lie in the $n_{1}+1$ cells $I_{1}, \ldots, I_{n_{1}+1}$ respectively. For each non-negative integer $r$, Let $Q_{n_{1}}(r)$ be the proportion of values among $r_{1}, \ldots, r_{n_{1}+1}$ which are equal to $r$. Then, in particular, we have $Q_{n_{1}}(0)=\frac{S_{0}}{n_{1}+1}$, the proportion of empty cells. 
Under the conditions (i) and (ii) of this section, J.R. Blum and L. Weiss in [1] prove that

$$
P\left[\begin{array}{ll}
\lim & \sup \\
\left(n_{1}, n_{2} ; p\right) & r \geq 0
\end{array}\left|Q_{1}(r)-Q(r)\right|=0\right]=1
$$

where $\lim$ denotes the limit as $\mathrm{n}_{1} \rightarrow \infty, \mathrm{n}_{2} \rightarrow \infty$ in such

$$
\left(n_{1}, n_{2} ; p\right)
$$

a way that $\mathrm{n}_{2} / \mathrm{n}_{1} \rightarrow \rho, \rho>0$, and

$$
Q(r)=\rho^{r} \int_{0}^{1} \frac{g^{2}(u)}{[\rho+g(u)]^{r+1}} d u
$$

where $g(u)$ is the derivative of $F_{2}\left(F_{1}^{-1}(u)\right)$, satisfying conditions (i) and (ii) of this section.

As a special case of (2.1) we have that

(2. 3)

$$
P\left[\begin{array}{l}
\lim \\
\left(n_{1}, n_{2} ; \rho\right)
\end{array}\left|Q_{n_{1}}(0)-Q(0)\right|=0\right]=1
$$

if $\left(F_{1}, F_{2}\right) \in G_{1}$, where we have now that

$$
Q(0)=\int_{0}^{1} \frac{g^{2}(u)}{[\rho+g(u)]} d u \text {. }
$$

It is also implied by (2. 3) that

$$
\lim _{\left(n_{1}, n_{2} ; \rho\right)} P\left(\left|Q_{n_{1}}(0)-Q(0)\right| \geq \epsilon\right)=0
$$

for any $\epsilon>0$, however small, if $\left(F_{1}, F_{2}\right) \in G_{1}$; that is $Q_{n_{1}}(0)=\frac{S_{0}}{n_{1}+1}$ converges in probability to $Q(0)$ (expression (2.4)).

Therefore, the test defined by (1.5) and (1.6) is consistent for testing any $\left(F_{1}, F_{2}\right) \in G_{0}$ against any $\left(F_{1}, F_{2}\right) \in G_{1}$ if 
(2.6)

$$
\int_{0}^{1} \frac{g^{2}(u)}{[\rho+g(u)]} d u>\frac{1}{1+\rho}
$$

where we recall from (1.4) that $1 / 1+p$ is the quantity to which $Q_{n_{1}}(0)=\frac{S_{0}}{n_{1}+1}$ converges in probability if $\left(F_{1}, F_{2}\right) \in G_{0}$.

The inequality of (2.6) is proved as follows. We have by Schwarz's inequality that

(2.7) $\int_{0}^{1} \frac{g^{2}(u) d u}{\rho+g(u)} \int_{0}^{1}(\rho+g(u)) d u>\left\{\int_{0}^{1} \frac{g(u)}{\sqrt{\rho+g(u)}} \sqrt{\rho+g(u)} d u\right\}$ that is

$$
\left(\int_{0}^{1} \frac{g^{2}(u) d u}{\rho+g(u)}\right)(\rho+1)>1
$$

which gives

$$
\int_{0}^{1} \frac{g^{2}(u) d u}{\rho+g(u)}>\frac{1}{1+\rho}
$$

if $g(u)$ differs from unity over a set of positive probability. This condition obtains if $\left(F_{1}, F_{2}\right) \in G_{1}$, since the derivatives of $F_{2}\left(F_{1}^{-1}(u)\right)=g(u)$ and $u$ are assumed to differ over a set of positive probability on $(0,1)$, and under this condition the above strict Schwarz inequality (2.7) holds. This completes the proof of the above theorem. 


\section{REFERENCES}

1. J.R. Blum and L. Weiss, Consistency of Certain TwoSample Tests. Ann. Math. Stat., Vol. 28 (1957), pp. 242-246.

2. S.S. Wilks, A Combinatorial Test for the Problem of Two Samples from Continuous Distributions. Proceedings of the Fourth Berkeley Symposium, Vol. I (1961), pp. 707-717.

3. S. S. Wilks, Mathematical Statistics. (Wiley, 1962).

4. M. Csorgo and Irwin Guttman, On the Empty Cell Test. Technometrics, Vol. 4, (1962), pp. 235-247.

McGill University and University of Wisconsin 